\title{
SG-ADVISER mtDNA: a web server for mitochondrial DNA annotation with data from 200 samples of a healthy aging cohort
}

\author{
Manuel Rueda* and Ali Torkamani*
}

\begin{abstract}
Background: Whole genome and exome sequencing usually include reads containing mitochondrial DNA (mtDNA). Yet, state-of-the-art pipelines and services for human nuclear genome variant calling and annotation do not handle mitochondrial genome data appropriately. As a consequence, any researcher desiring to add mtDNA variant analysis to their investigations is forced to explore the literature for mtDNA pipelines, evaluate them, and implement their own instance of the desired tool. This task is far from trivial, and can be prohibitive for nonbioinformaticians.

Results: We have developed SG-ADVISER mtDNA, a web server to facilitate the analysis and interpretation of mtDNA genomic data coming from next generation sequencing (NGS) experiments. The server was built in the context of our SG-ADVISER framework and on top of the MtoolBox platform (Calabrese et al., Bioinformatics 30(21): 3115-3117, 2014), and includes most of its functionalities (i.e., assembly of mitochondrial genomes, heteroplasmic fractions, haplogroup assignment, functional and prioritization analysis of mitochondrial variants) as well as a backend and a front-end interface. The server has been tested with unpublished data from 200 individuals of a healthy aging cohort (Erikson et al., Cell 165(4):1002-1011, 2016) and their data is made publicly available here along with a preliminary analysis of the variants. We observed that individuals over $\sim 90$ years old carried low levels of heteroplasmic variants in their genomes.

Conclusions: SG-ADVISER mtDNA is a fast and functional tool that allows for variant calling and annotation of human mtDNA data coming from NGS experiments. The server was built with simplicity in mind, and builds on our own experience in interpreting mtDNA variants in the context of sudden death and rare diseases. Our objective is to provide an interface for non-bioinformaticians aiming to acquire (or contrast) mtDNA annotations via MToolBox. SG-ADVISER web server is freely available to all users at https://genomics.scripps.edu/mtdna.
\end{abstract}

Keywords: Mitochondrial DNA, Annotation, Healthy aging, Heteroplasmy

\section{Background}

Next Generation Sequencing (NGS) technologies are revealing the complexity and richness of the human genome. While this revolution is blooming for nuclear DNA, much remains to be built out and matured for the 16,569 base pairs of the human mitochondrial genome (mtDNA), in particular for functional annotations of

\footnotetext{
* Correspondence: mrueda@scripps.edu; atorkama@scripps.edu The Scripps Translational Science Institute, Scripps Health, and The Scripps Research Institute, La Jolla, CA 92037, USA
}

disease associated variants. The ability to more routinely analyze mtDNA samples is crucial to establishing a more robust description of the specific genetic variants underlying mitochondrial disease [1], considered in tandem with disease causative variants in the nuclear genome [2]. In that regard, the existence of heteroplasmy (the presence of multiple alleles in an individual) in mtDNA demonstrates that the mitochondrial genome is a rich source of de-novo mutations potentially underlying many rare conditions [3-9]. For deleterious mutations, a 
minimum critical proportion of mutated copies (in the range of $60 \%-90 \%$ ) in the tissue(s) of relevance is necessary to display biochemical defects and phenotypic manifestation $[4,10]$. The proportion of mutated copies (a.k.a. mutation load) can differ among tissues and it might not be detectable, may be harder to detect, or may not be representative of the mutational load in the tissue of relevance when ascertained in a single tissue homogenate or blood sample [11]. Thus, we envision that in the future an individual may be sequenced several times (at the tissue level) to develop a more accurate picture of the expected severity and tissue specificity of a suspected mitochondrial disease. For all these reasons, there is a need for robust bioinformatic analysis of mtDNA variants.

Currently, there are many free services available for non-bioinformaticians seeking to carry out variant calling of nuclear variants from whole exome (WES) or whole genome (WGS) sequencing, e.g. Galaxy [12], GenePattern [13] or WEP [14] among others. However, with the exception of the newly published server by Weissensteiner et al. [15], there are few (or no) options for services amenable to non-bioinformaticians that appropriately deal with mitochondrial data. Thus, when a researcher performs WES/WGS analysis producing a negative result, and would like to expand the analysis to the mitochondrial genome, he or she will need to perform an exploration of the Linux command-line tools (i.e., MToolBox [16], MitoSeek [17], mit-o-matic [18]; note that MitoBamAnnotator [19] is no longer available) and make a decision according to that search. Per our own experience, comparison of these tools is far from trivial and we believe it results in a barrier, especially for labs that do not have the willingness or the expertise, to systematically analyze mtDNA variants. This barrier not only arises from the non-user friendly nature of command line tools themselves, but also from the process required to install command line pipelines. It often happens that to implement a computational pipeline, especially from academic software, one needs to co-install a plethora of accessory components, mostly softwarebased, but some also hardware-based. For instance, to create the reference sequence $k$-mers needed to install Gmap [20] within MToolBox, one needs 32GB of RAM, which is double what a typical non-specialized workstation usually contains. In our case, after testing a repertoire of packages we selected MToolBox v1.0 due to its robustness and richness of results (a comparative review of MToolBox performance was published elsewhere [15]). MToolBox is a highly automated bioinformatics pipeline that includes mtDNA assembling from WES or WGS data [21], heteroplasmic fraction detection with a related confidence interval, variant call format (VCF4.0) output, haplogroup assignment [22] and variant prioritization according to a disease score [23]. MToolBox is indeed a powerful tool, but in terms of data visualization only has a basic GUI (MSeqDR; https://mseqdr.org/mtoolbox.php). For this reason in our laboratory we developed an alternative way of visualizing MToolBox results that we incorporated to the analysis of our cases from the Molecular Autopsy [24] and IDIOM [25] studies.

In this light, we present SG-ADVISER mtDNA, a web server built on top of MToolBox, that attempts to simplify the human mitochondrial DNA variant calling, annotation and interpretation of variants. SG-ADVISER mtDNA utilizes SAM/BAM files and uses dynamic HTML web tables to display the results. The server was built having simplicity in mind, and is built upon our own experience in interpreting mitochondrial DNA mutations in the context of sudden death and rare diseases. Along with the server, we also provide individual level results for 200 healthy aging individuals that we analyzed and compared to reference cohorts. Our objective is to provide a simple alternative for non-bioinformaticians aiming to acquire (or contrast) mtDNA annotations via MToolBox.

\section{Implementation}

The SG-ADVISER mtDNA back-end was written in Perl 5. For the client-side operations, we used a responsive design web interface with HTML5 and JavaScript libraries. The entire core calculations are carried out by the MToolBox v1.0 suite as described elsewhere [16], as well as with in-house scripts (see Additional file 1: Text T1). The reference genome used is the Reconstructed Sapiens Reference Sequence (RSRS) [26].

\section{Input data}

The server functions in two modes, "individual sample" and "cohort". In the former, the user can upload a single SAM/BAM file, whereas in the latter the user can upload a whole directory consisting of multiple SAM or BAM files. Cohort mode is a good choice for family pedigrees or small-size populations, as the results for each variant will be shown aggregate in one line. We deliberately restricted the input to be SAM/BAM files, knowing that they have become a de facto standard for sharing sequence data. Rather than uploading the whole WES/ WGS alignment file, we ask the user to upload only the mitochondrial DNA reads. This is the only "technical" step that needs to be performed prior to submission and can be easily achieved with SAMtools [27], as described on the help section of the server. This way, we avoid the unnecessary transfer of large data files over the network, much of which will not be processed anyway. Note that the server will re-align the reads with Gmap, so the alignment in the original file is just required to isolate reads mapping to the mtDNA genome. All the data 
transfer is performed securely through an SSL certificate. Uploaded SAM/BAM files are deleted after job completion and results are kept for a week, after that all the data are permanently deleted. Apart from the data upload, there are three optional parameters for the user: i) a text field for an email address to get notified when the job finishes, ii) an option to set the job to private so that only the user who sent the job will have the link to the results (note that an email is mandatory when this option is selected), and iii) a text field to change the default job identifier. Apart from a standalone calculation, we envision that some users may wish to use the Linux command line to launch multiple jobs. For that purpose, in the help section we provided scripts for web services that will avoid the necessity for "screen scraping" of HTML.

\section{Output data}

Upon submission, each job is sent to a PBS queue system installed in a local dedicated server. The hardware consists of $1 \mathrm{x}$ Intel Xeon CPU E5-2630 V4 2.2GHz with 64GB of RAM and 16 TB of HDD, capable of running 20 simultaneous threads. The alignment step with Gmap-gsnap [20] benefits from parallelization, thus, in a compromise between speed and capacity we set the number of threads per sample to 4 . With this set-up, analysis of one sample at 2200X coverage takes $\sim 2 \mathrm{~min}$ to complete. At full capacity the server will support $\sim 150$ samples per hour ( 3600 samples per day). Once a job is submitted to the queue, the user is redirected to the status page that contains information about the completion of submitted jobs. When a job is finished, the results page becomes available via a link. In the results page, all the prioritized variants coming from MToolBox are displayed, as well as appended information that we extract from the final VCF files (i.e., heteroplasmic fraction, depth and genotype information). The HTML table has several functionalities, such as URL links to external databases, sorting, search (regular expressions allowed), rearranging of columns, etc. All the results can be downloaded as text files by clicking in the corresponding link in the page. The server includes a pre-computed example as well as the 200 individual Wellderly samples, plus a help page with extended documentation on the technical details.

\section{Results and discussion}

\section{Analysis of the healthy aging cohort}

The healthy aging cohort (a.k.a. the Wellderly) is defined as individuals who were $>80$ years old with no chronic diseases and who were not taking chronic medications (see full criteria of inclusion at [28]). Here we analyzed unpublished data from 200 Wellderly individuals who had their WGS sequenced with the Illumina Moleculo technology [29]. For each individual, we extracted mtDNA reads from WGS BAM files to create mtDNA-only BAM files that were later submitted to our server. We set up the server so that all the data could be browsed and downloaded (see help page).

Apart from allowing visualization of the individual level data on the browser, given that mtDNA has been associated with aging in the literature $[2,6,30-40]$, we carried out basic statistics on the abundance and distribution of variants within the mitochondrial genome. We would like to emphasize that our objective with this publication is not to perform a comprehensive casecontrol study, but rather to make the data publicly available along with the server.

\section{1) Effect of depth of coverage on the number of variants}

First, we investigated the effect of coverage (i.e., number of reads per position) on the number of detected mtDNA variants. The DNA for all 200 individuals was extracted from peripheral blood and the average depth (per position) after the sequencing was $2281 \pm 594$ reads per sample (min value: 1037, max value: 5166 ). The coverage showed remarkable variability, despite the fact that all samples were sequenced under similar conditions. It is worth mentioning that the disparity in coverage did not stem from differences in the amount of DNA loaded in the plate (see Additional file 1: Figure S1), nor it is correlated to the age of the individuals (see Additional file 1: Figure S2). It is unknown whether this change in DNA abundance is due to actual differences in the number of chromosomal copies, or if it is due to other sample preparation issues during the sequencing process. In any case, even with coverages that exceed 1000X, a common concern is recognizing to what extent the depth affects the capacity to capture essential variants. Figure 1 shows a scatterplot of the total number of variants with respect to the variants having a heteroplasmic fraction $>0.3$ (see discussion about the threshold selection at Additional file 1: Figure S3 and [41, 42]). With the exception of three samples, all others consisted of $<500$ total variants, the majority having $<400$ (median number of variants per individual was 116.5 , interquartile range: $88-157$, min value: 34, max value: 1988). Samples with an average depth $>$ 2500X consistently contained more total detected variants than those with $<2500 \mathrm{X}$, but the majority of these "additional" variants had extremely low heteroplasmic fractions (see Fig. 1), and therefore many of these variants are potentially noise, sequencing errors, or variant calling artifacts [42]. Increasing the depth of coverage to $>1000 \mathrm{X}$ did not affect the number of variants detected at HF $>0.3$. In other words, a minimum depth of 300 reads for the alternative allele was sufficient to capture all variants considered in this analysis and variations in depth of coverage did not influence our results. 


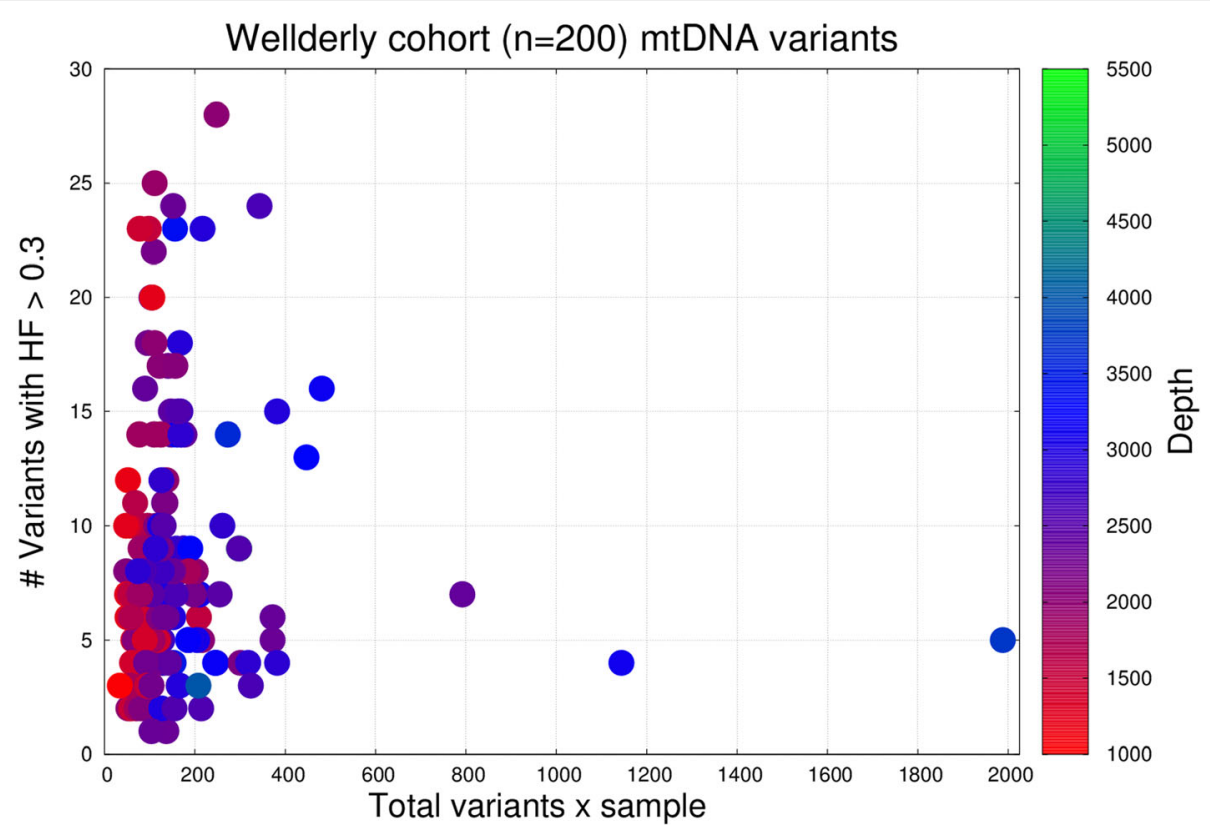

Fig. 1 Scatter plot showing the total number of mitochondrial DNA (mtDNA) variants per sample vs. the number of variants with heteroplasmic fraction (HF) > 0.3 in 200 Wellderly individuals. Each dot represents 1 individual. The colouring scheme represents the average (per sample) depth of coverage for mtDNA variants

\section{2) Distribution of pathogenic variants across the mtDNA genome}

The total number of mitochondrial DNA variants found in all 200 individuals was 30,445 (see Additional file 1: Figure S3). From these, 550 (1.8\%) were insertions or deletions, the rest being single nucleotide polymorphisms (SNPs). Four thousand nine hundred sixteen out of the 30,445 (16\%) were synonymous variants. When filtered by $\mathrm{HF}>0.3$ the total number of variants was 1654 , which yielded a median number of 7 heteroplasmic variants per sample (interquartile range: $5-9$, min value: 1 , max value: 28 ).

To investigate the distribution of heteroplasmic variants across the mitochondrial genome, we grouped heteroplasmic variants according to their locus and built a histogram with their frequencies (see Fig. 2a). For comparison purposes, we also included the results obtained with 32,059 samples from GenBank (25 June 2016 version) downloaded from the Mitomap database [43, 44]. Despite the difference in cohort size, ancestry, sequencing technology, etc. the amount of heteroplasmic variation per locus seems to be stable in both cohorts. The only exceptions to this rule were the genes $M T-C Y B$ (mitochondrially encoded cytochrome b) and MT-RNR1 (mitochondrially encoded $12 \mathrm{~S}$ RNA), both accumulating a larger number of variants in GenBank cohort [45]. Most heteroplasmic variants tend to accumulate in the $M T-D L O O P$ [46], followed by the $M T-N D[X]$ complexes (mitochondrially encoded NADHs complexes). The MT-
$D L O O P$ is the longest noncoding region in vertebrate mtDNA and contains the $\mathrm{H}$-strand replication origin. Two MT-DLOOP regions (hypervariable regions HVR1 and HVR2) are known for accumulating more variants than anywhere else in the mitochondrial genome. The $M T-N D[X]$ regions are the largest coding loci in the mtDNA genome and, thus, an excess of the absolute number of mitochondrial mutations is expected. On the other hand, tRNA genes are small and should accumulate fewer mutations in total. This is indeed what we observed (see Fig. 2b) with the exception of $M T-D L O O P$ region.

After checking variants at the locus level, we sought to investigate specific variants associated with disease in the literature. For this purpose, we selected all Wellderly variants with $\mathrm{HF}>0.3$ that had been associated with disease in the MitoMap database, and compared their abundance with respect to the GenBank cohort, as well as to that in 1000 Genomes (1000G) cohort [47] (see Table 1). Again, despite the heterogeneity of the data, we found overall concordance. GenBank had spikes in three particular positions, in 3010A (rs3928306) a SNP for which we could not determine anything out of the ordinary other than association studies to eye diseases, and in $11467 \mathrm{G}$ and $12372 \mathrm{~A}$, both being the only synonymous substitutions found in the list. The total number of pathogenic variants for the Wellderly cohort was 79 (0.39 per sample), whereas for the GenBank was 39,688 (1.24 per sample). When we excluded the 3 variants with 

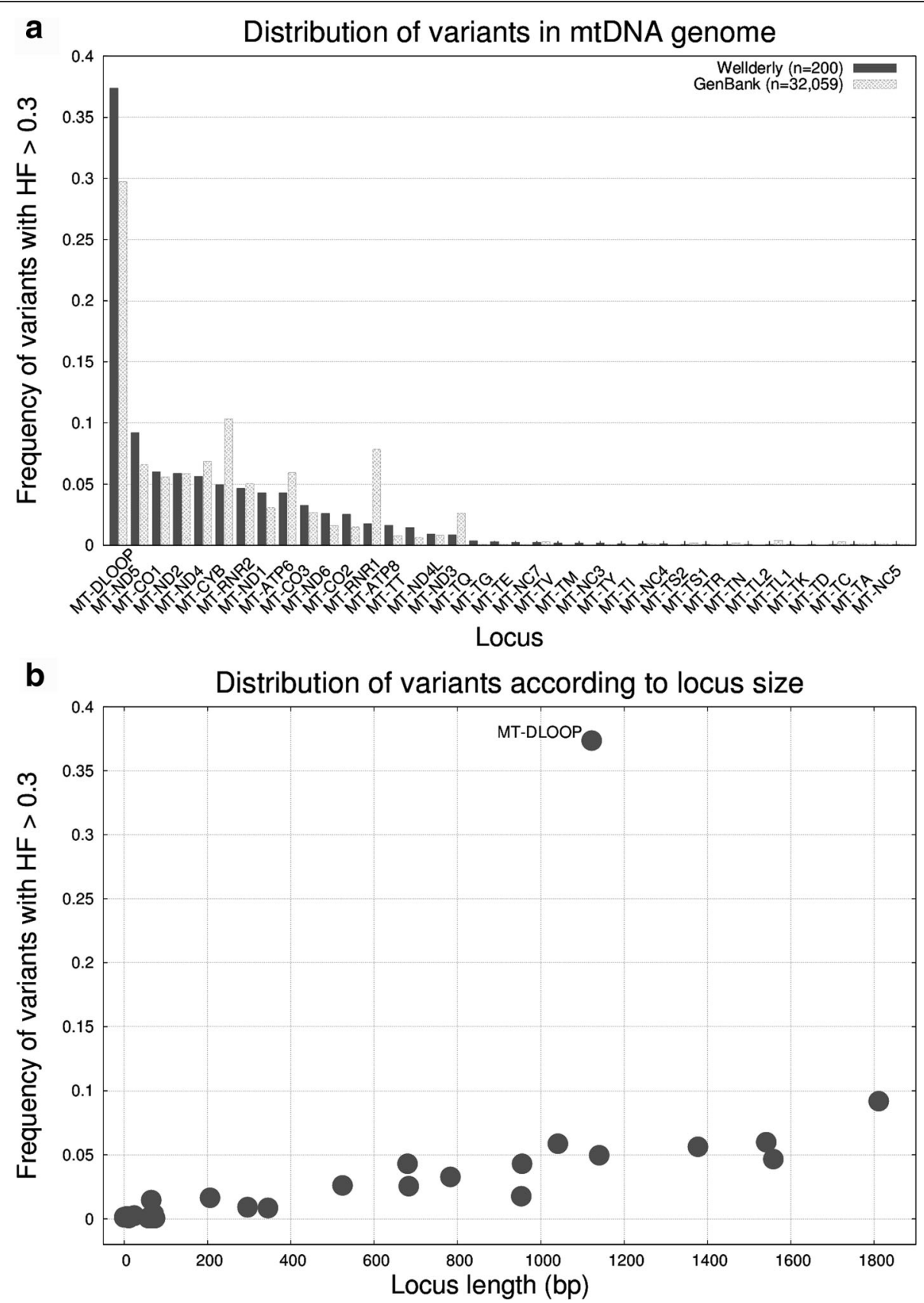

Fig. 2 a Histogram showing the distribution of the Wellderly cohort heteroplasmic variants ( $H F>0.3$ ) across the mitochondrial DNA genome. The loci are numerically sorted according the number of variants. As a reference, we display also values for the GenBank cohort. b Scatter plot of the locus length vs. the frequency of heteroplasmic mutations at the locus. Note that the number of mutations correlates almost linearly with the locus size, except for the MT-DLOOP

large excess (see above) we ended up with 76 (0.38 per sample) vs. 25,910 (0.81 per sample). This two fold increase in the GenBank cohort might be due to differences in the level of heteroplasmy reported, intrinsic errors of GenBank data [45], or enrichment of diseased individuals in the GenBank database. In terms of locus distribution, most of the pathogenic mutations (59\%) fell in the MT-DLOOP [46] and a very few in the tRNA genes (except for MT-TE and MT-TT).

\section{3) Phenotypic effects contributing to high heteroplasmic levels}

Finally, we investigated the amount of heteroplasmy per sample versus several self-reported parameters/conditions 
Table 1 Heteroplasmic variants (HF > 0.3) present in the Wellderly cohort associated with disease in the MitoMap database

\begin{tabular}{|c|c|c|c|c|c|c|c|c|}
\hline \multirow[t]{2}{*}{ Variant } & \multirow[t]{2}{*}{ Locus } & \multirow[t]{2}{*}{ Aa Change } & \multirow[t]{2}{*}{ MitoMap Associated Disease(s) } & \multirow{2}{*}{$\begin{array}{l}\text { Wellderly } \\
\text { AC }\end{array}$} & \multirow{2}{*}{$\begin{array}{l}n=200 \\
A F\end{array}$} & \multirow{2}{*}{$\begin{array}{l}\mathrm{GB} \\
\mathrm{AC}\end{array}$} & \multirow{2}{*}{$\begin{array}{l}n=32,059 \\
A F\end{array}$} & \multirow{2}{*}{$\begin{array}{l}1000 \mathrm{G} \\
\mathrm{AF}\end{array}$} \\
\hline & & & & & & & & \\
\hline $961 C$ & MT-RNR1 & & DEAF possibly LVNC-associated & 2 & 0.010 & 317 & 0.010 & 0.0066 \\
\hline $961 G$ & MT-RNR1 & & Possibly DEAF-associated & 2 & 0.010 & 123 & 0.004 & 0.0008 \\
\hline $2352 \mathrm{C}$ & MT-RNR2 & & Possibly LVNC-associated & 1 & 0.005 & 839 & 0.026 & 0.0737 \\
\hline $2361 \mathrm{~A}$ & MT-RNR2 & & Possibly LVNC-associated & 1 & 0.005 & 107 & 0.003 & 0.0058 \\
\hline $3010 \mathrm{~A}$ & MT-RNR2 & & Cyclic Vomiting Syndrome with Migraine & 1 & 0.005 & 5046 & 0.157 & 0.1043 \\
\hline $3796 G$ & MT-ND1 & T164A & Adult-Onset Dystonia & 1 & 0.005 & 174 & 0.005 & 0.0029 \\
\hline $4454 C$ & MT-TM & & Possible contributor to mito dysfunction / HTA & 1 & 0.005 & 185 & 0.006 & 0.0041 \\
\hline $5913 \mathrm{~A}$ & MT-CO1 & D4N & Prostate Cancer/hypertension & 2 & 0.010 & 241 & 0.008 & 0.0029 \\
\hline $6253 C$ & MT-CO1 & M117T & Prostate Cancer & 2 & 0.010 & 355 & 0.011 & 0.0103 \\
\hline $6261 \mathrm{~A}$ & MT-CO1 & A120T & Prostate Cancer / LHON & 1 & 0.005 & 176 & 0.005 & 0.0054 \\
\hline $6489 A$ & MT-CO1 & L196l & Therapy-Resistant Epilepsy & 2 & 0.010 & 67 & 0.002 & 0.0008 \\
\hline $7041 \mathrm{~A}$ & MT-CO1 & V380I & Prostate Cancer & 1 & 0.005 & 5 & 0.000 & 0.0004 \\
\hline 8393Т & MT-ATP8 & P10S & Reversible brain pseudoatrophy & 3 & 0.015 & 127 & 0.004 & 0.0021 \\
\hline $9055 \mathrm{~A}$ & MT-ATP6 & $\mathrm{A} 177 \mathrm{~T}$ & PD protective factor & 2 & 0.010 & 1581 & 0.049 & 0.0189 \\
\hline $10454 C$ & MT-TR & & DEAF helper mut & 1 & 0.005 & 118 & 0.004 & 0.0033 \\
\hline $11467 G$ & MT-ND4 & syn & Altered brain $\mathrm{pH}$ & 1 & 0.005 & 4213 & 0.131 & 0.0717 \\
\hline $12372 \mathrm{~A}$ & MT-ND5 & syn & Altered brain $\mathrm{pH}$ & 1 & 0.005 & 4519 & 0.141 & 0.0829 \\
\hline $14687 G$ & MT-TE & & Mito myopathy w respiratory failure & 4 & 0.020 & 211 & 0.007 & 0.0074 \\
\hline 15927A & MT-TT & & Multiple Sclerosis / DEAF1555 inc. penetrance & 3 & 0.015 & 317 & 0.010 & 0.007 \\
\hline $16093 C$ & MT-DLOOP & & Cyclic Vomiting Syndrome & 14 & 0.070 & 3982 & 0.124 & 0.0567 \\
\hline $16176 \mathrm{~T}$ & MT-DLOOP & & Cyclic Vomiting Syndrome with Migraine & 2 & 0.010 & 523 & 0.016 & 0.0029 \\
\hline $16183 \mathrm{C}$ & MT-DLOOP & & Melanoma patients & 12 & 0.060 & 9632 & 0.300 & 0.0869 \\
\hline $16192 T$ & MT-DLOOP & & Melanoma patients & 11 & 0.055 & 3087 & 0.096 & 0.0488 \\
\hline $16270 T$ & MT-DLOOP & & Melanoma patients & 8 & 0.040 & 3111 & 0.097 & 0.0562 \\
\hline
\end{tabular}

As a reference, we also display GenBank and 1000G cohorts. Note that SG-ADVISER mtDNA uses RSRS (Reconstructed Sapiens Reference Sequence) numbering schema whereas data in Mitomap uses Cambridge Reference Sequence (rCRS). We made sure that the numbering schema was equivalent for the variants studied. Acronyms: GB (GenBank), 1000G (1000 Genomes), AF (allele frequency), AC (allele count)

such as age, body mass index, smoking status, etc. In Fig. 3 we show a side-by-side histogram of the frequency of variants with $\mathrm{HF}>0.3$ for males $(n=69)$ and females $(n=131)$. Both genders behave similarly in terms of variant distribution, hence, gender does not appear to be affecting the amount of heteroplasmic variants. In Fig. 4a we compared the number of heteroplasmic variants with respect to age for females and males, and we also did not observed that aging caused an increase in the number of heteroplasmic variants [37] nor is there an interaction between aging and gender on the rate of heteroplasmic variants. Instead, and contrary to our intuition, we observed that individuals over the age of $\sim 90$ tended to have a lower number of heteroplasmic variants. By inspecting the 30 variants present in the 5 women older than 100 years old, we observed that 25 (83\%) were SNPs, that 13 (43.3\%) were synonymous, that only 1 variant $(3 \%)$ was associated with disease (m.15077G>A, DEAF: maternally inherited nonsyndromic hearing loss) in the Mitomap database, that
$1 / 3(33 \%)$ of the variants were rare (allele frequency $<0.05$ in $1000 \mathrm{G})$, that the HF were high ( $81 \%$ had HF $>0.8)$, and that $16(53 \%)$ were in the MT-DLOOP region $(87 \%, 37 \%$, $11 \%, 18 \%, 83 \%$ and $37 \%$ respectively when taking into account the 200 individuals). In fact, a moderate linear relationship (uphill) was observed between the number of heteroplasmic variants in the MT-DLOOP and the total number of variants (see Additional file 1: Figure S4), which suggests that the MT-DLOOP integrity might play a role in, or be a surrogate for, the overall mutational rate. Taking the above observations together, we hypothesize that inheriting a "stable" mtDNA genome might provide an optimum metabolic efficiency that, as a result, contributes to disease prevention. Based on our data, we cannot determine whether the tendency to have a lower number of heteroplasmic variants after age $\sim 90$ is due to sample size, decrease of cell division rate/metabolism associated with age, or if it is due to protective genetic mechanisms. For nuclear DNA, somatic mutations in the context of 


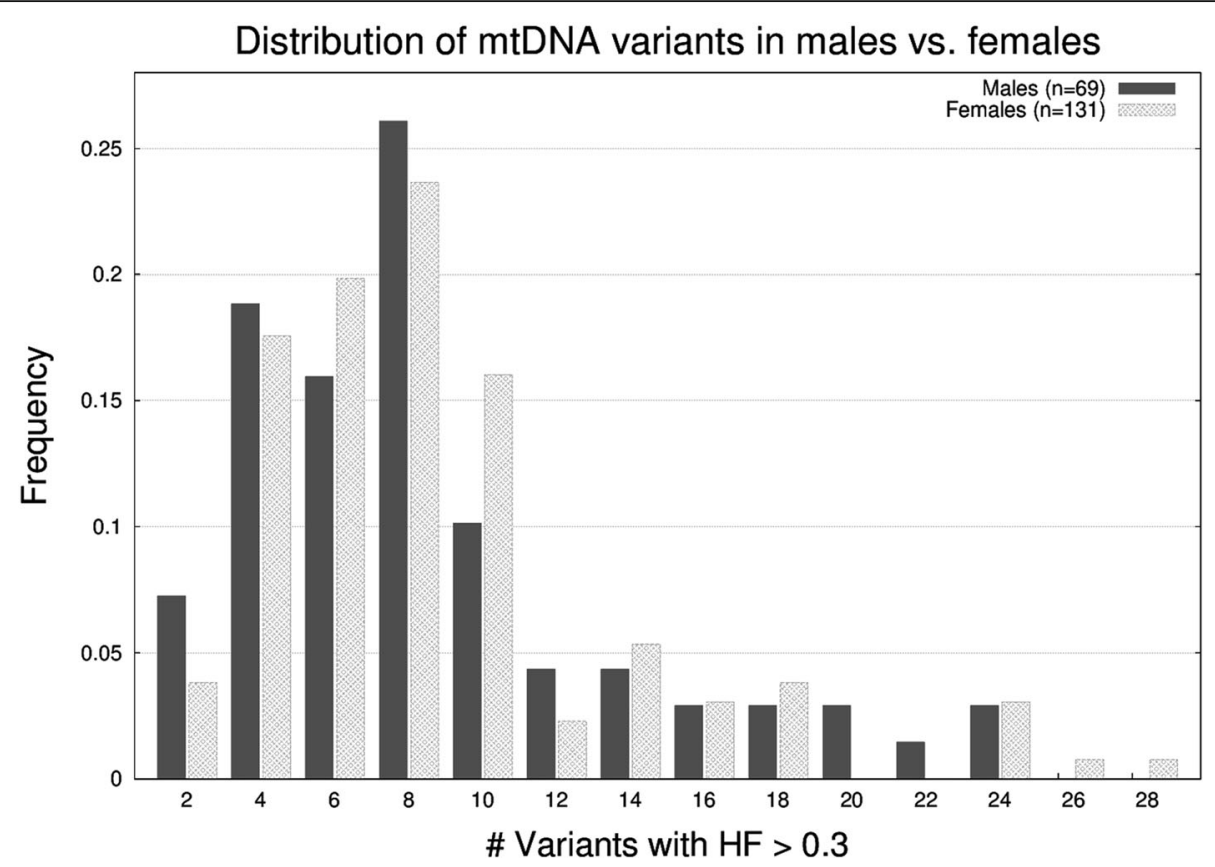

Fig. 3 Histogram of the distribution of mitochondrial DNA heteroplasmic variants (HF > 0.3) according to gender in 200 Wellderly individuals

clonal hematopoiesis have been show to increase with age [48] but recently it has been show that the amount of somatic mutation on induced pluripotent stem cells (iPSC) decreases after age 90 [49].

Lastly, for all other parameters we reported median values, interquartile ranges and $p$-values from a MannWhitney U test (see Table 2) used to test for association with heteroplasmic levels. After the Bonferroni correction, we did not observe any association between the number of heteroplasmic variants and any of the parameters studied. The spreadsheet consisting of all the phenotypic information that we compiled is available as Additional file 2: Table S1.

\section{Conclusions}

We have developed a web tool named SG-ADVISER mtDNA that allows for efficient variant calling, annotation and priorization of variants from human mtDNA
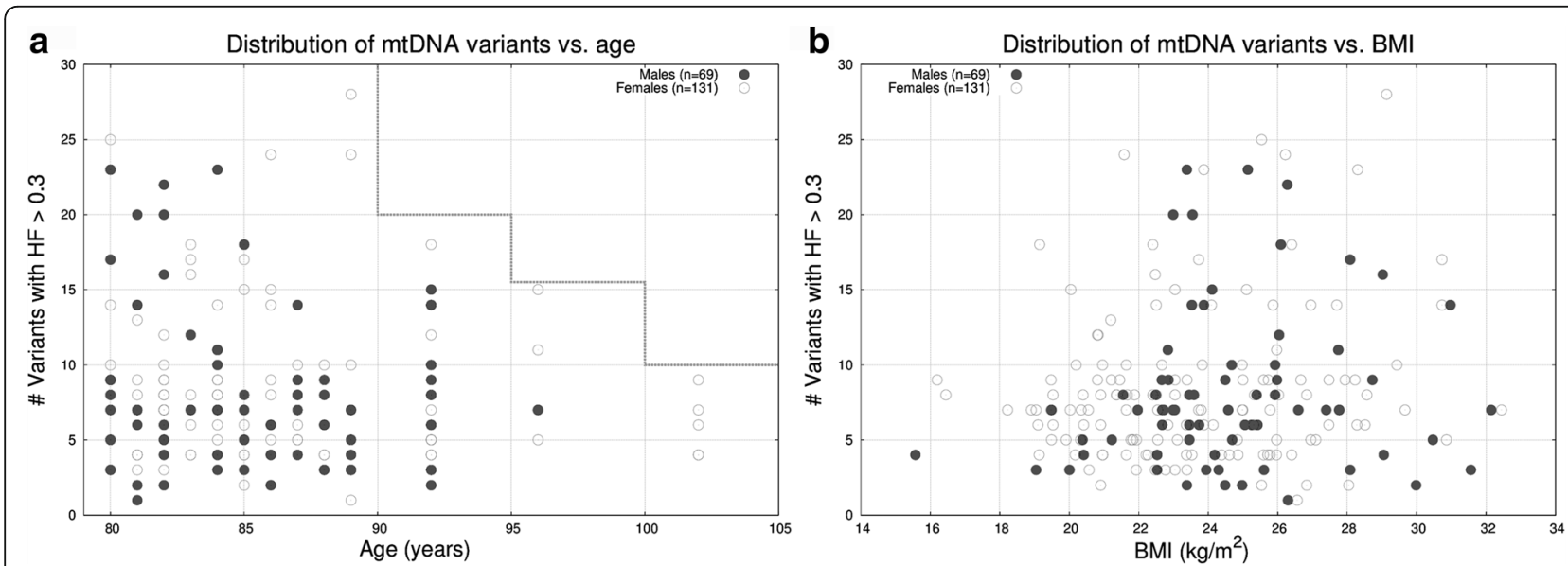

Fig. 4 Scatter plots of two phenotypic parameters vs. the number of mitochondrial DNA variants with heteroplasmic fraction (HF) $>0.3$ in the Wellderly cohort: a age vs. number of variants; note the decrease in number after the age of 90 . b Body Mass Index (BMI) vs. number of variants, no relation found 
Table 2 Median values, [interquartile ranges (sample size)] and Mann-Whitney $U$ test $p$-values (uncorrected and Bonferroni corrected (BF)) to test for association between 14 self-reported conditions and the number heteroplasmic variants (HF >0.3) present in the Wellderly cohort

\begin{tabular}{|c|c|c|c|c|}
\hline Condition & $\begin{array}{l}\text { Yes } \\
\text { Median [IQR (n)] }\end{array}$ & $\begin{array}{l}\text { No } \\
\text { Median [IQR (n)] }\end{array}$ & $\begin{array}{l}\text { Mann-Whitney } U \text { test } \\
p \text {-value }\end{array}$ & $\begin{array}{l}\text { Mann-Whitney } U \text { test } \\
p \text {-value (BF) }\end{array}$ \\
\hline Blad_control & $7.0[5-8(6)]$ & $7.0[5-10(192)]$ & 0.5974 & 1.0000 \\
\hline Bph & 7.0 [3-9 (24)] & 7.0 [5-9 (174)] & 0.7815 & 1.0000 \\
\hline Copd_asthma & $6.0[3-9(7)]$ & 7.0 [5-9 (193)] & 0.4505 & 1.0000 \\
\hline Depr_anx & 7.0 [3-9 (12)] & $7.0[5-9(188)]$ & 0.5750 & 1.0000 \\
\hline Dyslipidemia & $8.0[6-12(46)]$ & $7.0[4-9(154)]$ & 0.0833 & 1.0000 \\
\hline Gerd & 7.0 [6-9 (19)] & 7.0 [5-9 (181)] & 0.4879 & 1.0000 \\
\hline Glaucoma & $7.0[4-11(30)]$ & $7.0[5-9(170)]$ & 0.8320 & 1.0000 \\
\hline Hrt & $8.5[7-10(12)]$ & 7.0 [5-9 (188)] & 0.0682 & 0.9549 \\
\hline Hyperten & $7.5[5-10(80)]$ & $7.0[4-9(120)]$ & 0.0399 & 0.5585 \\
\hline Hypothyroid & 7.0 [5-10 (39)] & 7.0 [5-9 (161)] & 0.7091 & 1.0000 \\
\hline Macular_degen & 7.0 [5-9 (15)] & $7.0[5-9(185)]$ & 0.9132 & 1.0000 \\
\hline Osteoarth & $7.0[5-10(87)]$ & 7.0 [5-9 (113)] & 0.3987 & 1.0000 \\
\hline Smoking_hist & 7.0 [4-9 (89)] & $7.0[5-10(111)]$ & 0.1922 & 1.0000 \\
\hline Take_meds & $7.0[5-9(161)]$ & 7.0 [5-9 (39)] & 0.7356 & 1.0000 \\
\hline
\end{tabular}

Labels: Blad_control: Bladder control problems; Bph: Benign prostatic hyperplasia; Depr_anx: Depression or anxiety; Gerd: Gastroesophageal reflux disease; Hrt: Hormone replacement therapy, Hyperten: Hypertension; Macular_degen: Macular degeneration; Osteoarth: Osteoarthritis; Smoking_hist: Smoking history; Take_meds: Currently taking medications

SAM/BAM files. The web server has been tested with 200 unpublished mtDNA genomes from a healthy aging cohort and the data has been made public available here. The distribution of heteroplasmic variants in the Wellderly cohort did not substantially differ from that in GenBank or $1000 \mathrm{G}$ cohorts. Pending replication, we observed that individuals over the age of $\sim 90$ tend to have a low number of heteroplasmic variants in their mitochondrial genomes.

\section{Additional files}

Additional file 1: Figures S1, S2, S3, S4 and Text T1. (DOCX 773 kb)

Additional file 2: A spreadsheet consisting of all the phenotypic information for the 200 Wellderly individuals. (XLSX 86 kb)

\section{Abbreviations}

1000G: 1000 Genomes; BAM: Binary SAM format; CPU: Central processing unit; DEAF: Maternally inherited nonsyndromic hearing loss; DNA: Deoxyribonucleic acid; GUI: Graphical user interface; HDD: Hard disk drive; HF: Heteroplasmic fraction; HTML: Hypertext markup language; HVR: Hypervariable region; mtDNA: Mitochondrial DNA; NGS: Next generation sequencing; PBS: Portable batch system; RAM: Random access memory; RSRS: Reconstructed sapiens reference sequence; SAM: Sequence alignment/map format; SNP: Single nucleotide polymorphism; tRNA: Transfer ribonucleic acid; VCF: Variant call format; WES: Whole exome sequencing; WGS: Whole genome sequencing

\section{Acknowledgements}

We thank Illumina Inc. for performing the sequencing, Alex Lippman for technical support and Emily Spencer for helping us with the Additional file 2.

\section{Funding}

This work was supported by NIH grants U54GM114833, U01 HG006476 and 5 UL1 RR025774. The funding agency played no role in the design or the conclusion of this study.

Availability of data and materials

SG-ADVISER mtDNA software as well as the Wellderly dataset can be accessed at https://genomics.scripps.edu/mtdna.

- Operating system(s): Platform independent

- Programming languages: Perl 5, HTML5, JavaScript.

- License: GNU GPL

- Any restrictions to use by non-academics: None.

\section{Authors' contributions}

MR designed, implemented and tested the web server, analysed the cohorts and wrote the manuscript. AT obtained the funding and revised the manuscript. Both authors read and approved the final manuscript.

Ethics approval and consent to participate

The Healthy Elderly Active Longevity Cohort Study (IRB-13-6142) was approved by the Scripps Institutional Review Board in July 2007. Participants consented to broad sharing of anonymized phenotypic data.

\section{Competing interests}

The authors declare that they have no competing interest.

\section{Publisher's Note}

Springer Nature remains neutral with regard to jurisdictional claims in published maps and institutional affiliations. 
Received: 7 April 2017 Accepted: 31 July 2017

Published online: 18 August 2017

\section{References}

1. Gorman $G$, Chinnery $P$, Dimauro S, Hirano $M$, Koga $Y$, Mcfarland $R$, Suomalainen A, Thorburn D, Zeviani M, Turnbull D. Mitochondrial diseases. Nature Reviews Disease Primers. 2016;2:16080.

2. Taylor R, Turnbull D. Mitochondrial Dna mutations in human disease. Nat Rev Genet. 2005;6(5):389-402.

3. Coller H, Khrapko K, Bodyak ND, Nekhaeva E, Herrero-Jimenez P, Thilly WG. High frequency of Homoplasmic mitochondrial Dna mutations in human tumors can be explained without selection. Nat Genet. 2001;28(2):147-50.

4. Rossignol R, Faustin B, Rocher C, Malgat M, Mazat JP, Letellier T. Mitochondrial threshold effects. The Biochemical Journal. 2003;370(Pt 3):751-62.

5. Tuppen H, Blakely E, Turnbull D, Taylor R. Mitochondrial Dna mutations and human disease. Biochim Biophys Acta. 2010;1797(2):113-28.

6. Stewart J, Larsson NG. Keeping Mtdna in shape between generations. PLoS Genet. 2014:10(10)

7. Ye K, Lu J, Ma F, Keinan A, Gu Z. Extensive Pathogenicity of mitochondrial Heteroplasmy in healthy human individuals. Proc Natl Acad Sci U S A. 2014; 111(29):10654-9.

8. Stewart J, Chinnery P. The dynamics of mitochondrial Dna Heteroplasmy: implications for human health and disease. Nat Rev Genet. 2015;16(9):530-42.

9. Chinnery P, Howell N, Lightowlers R, Turnbull DM. Melas and Merrf. The relationship between maternal mutation load and the frequency of clinically affected offspring. Brain : A Journal Of Neurology. 1998;121(Pt 10):1889-94.

10. Wong L. Diagnostic challenges of mitochondrial Dna disorders. Mitochondrion. 2007;7(1-2):45-52

11. Yao Y, Kajigaya S, Young NS. Mitochondrial Dna mutations in single human blood cells. Mutat Res. 2015;779:68-77.

12. Afgan E, Baker D, Van Den Beek M, Blankenberg D, Bouvier D, Cech M, Chilton J, Clements D, Coraor N, Eberhard C, et al. The galaxy platform for accessible, reproducible and collaborative biomedical analyses: 2016 update. Nucleic Acids Res. 2016:44(W1):W3-W10.

13. Reich M, Liefeld T, Gould J, Lerner J, Tamayo P, Mesirov JP. Genepattern 2.0. Nat Genet. 2006:38(5):500-1.

14. D'antonio M, D'onorio De Meo P, Paoletti D, Elmi B, Pallocca M, Sanna N, Picardi E, Pesole G, Castrignano T. Wep: a high-performance analysis pipeline for whole-exome data. Bmc Bioinformatics. 2013;14(Suppl 7):S11.

15. Weissensteiner $H$, Forer L, Fuchsberger C, Schopf B, Kloss-Brandstatter A, Specht G, Kronenberg F, Schonherr S. Mtdna-server: next-generation sequencing data analysis of human mitochondrial Dna in the cloud. Nucleic Acids Res. 2016:44(W1):W64-9.

16. Calabrese C, Simone D, Diroma MA, Santorsola M, Gutta C, Gasparre G, Picardi E, Pesole G, Attimonelli M. Mtoolbox: a highly automated pipeline for Heteroplasmy annotation and prioritization analysis of human mitochondrial variants in high-throughput sequencing. Bioinformatics. 2014;30(21):3115-7.

17. Guo Y, Li J, Li Cl, Shyr Y, Samuels DC. MitoSeek: extracting mitochondria information and performing high-throughput mitochondria sequencing analysis. Bioinformatics. 2013;29(9):1210-1.

18. Vellarikkal S, Dhiman H, Joshi K, Hasija Y, Sivasubbu S, Scaria V. Mit-O-Matic: a comprehensive computational pipeline for clinical evaluation of mitochondrial variations from next-generation sequencing datasets. Hum Mutat. 2015;36(4):419-24.

19. Zhidkov I, Nagar T, Mishmar D, Rubin E. Mitobamannotator: a web-based tool for detecting and annotating Heteroplasmy in human mitochondrial Dna sequences. Mitochondrion. 2011;11(6):924-8.

20. Wu T, Watanabe C. Gmap: a genomic mapping and alignment program for Mrna and Est sequences. Bioinformatics. 2005:21(9):1859-75.

21. Picardi E, Pesole G. Mitochondrial genomes gleaned from human wholeexome sequencing. Nat Methods. 2012;9(6):523-4.

22. Rubino F, Piredda R, Calabrese F, Simone D, Lang M, Calabrese C, Petruzzella V, Tommaseo-Ponzetta M, Gasparre G, Attimonelli M. Hmtdb, a genomic resource for mitochondrion-based human variability studies. Nucleic Acids Res. 2012:40(Database Issue):D1150-9.

23. Santorsola M, Calabrese C, Girolimetti G, Diroma M, Gasparre G, Attimonelli M. A multi-parametric workflow for the prioritization of mitochondrial Dna variants of clinical interest. Hum Genet. 2016;135(1):121-36.
24. Torkamani A, Muse E, Spencer E, Rueda M, Wagner G, Lucas JR, Topol EJ. Molecular autopsy for sudden unexpected death. Jama-J Am Med Assoc. 2016;316(14):1492-4

25. Bloss C, Zeeland A, Topol S, Darst B, Boeldt D, Erikson G, Bethel K, Bjork R, Friedman J, Hwynn N, et al. A genome sequencing program for novel undiagnosed diseases. Genet Med. 2015;17(12):995-1001.

26. Behar D, Van Oven M, Rosset S, Metspalu M, Loogvali E, Nm S, Kivisild T, Torroni A, Villems R. A "Copernican" reassessment of the human mitochondrial Dna tree from its root. Am J Hum Genet. 2012;90(4):675-84.

27. Li H, Handsaker B, Wysoker A, Fennell T, Ruan J, Homer N, Marth G, Abecasis $G$, Durbin R. Genome project data processing $S$ : the sequence alignment/map format and Samtools. Bioinformatics. 2009;25(16):2078-9.

28. Erikson G, Bodian D, Rueda M, Molparia B, Scott E, Scott-Van Zeeland A, Topol S, Wineinger N, Niederhuber J, Topol E, et al. Whole-genome sequencing of a healthy aging cohort. Cell. 2016;165(4):1002-11.

29. Kuleshov V, Xie D, Chen R, Pushkarev D, Ma Z, Blauwkamp T, Kertesz M, Snyder M. Whole-genome Haplotyping using long reads and statistical methods. Nat Biotechnol. 2014;32(3):261-6.

30. Kang E, Wang $X$, Tippner-Hedges $R$, Ma H, Cd F, Gutierrez N, Lee $Y$, Van Dyken C, Ahmed R, Li Y, et al. Age-related accumulation of somatic mitochondrial Dna mutations in adult-derived human Ipscs. Cell Stem Cell. 2016;18(5):625-36

31. Kennedy S, Salk J, Schmitt M, Loeb L. Ultra-sensitive sequencing reveals an age-related increase in somatic mitochondrial mutations that are inconsistent with oxidative damage. PLoS Genet. 2013;9(9):E1003794.

32. Sevini F, Giuliani C, Vianello D, Giampieri E, Santoro A, Biondi F, Garagnani $P$, Passarino G, Luiselli D, Capri M, et al. Mtdna mutations in human aging and longevity: controversies and new perspectives opened by high-throughput technologies. Exp Gerontol. 2014;56:234-44.

33. Sun $N$, Youle R, Finkel T. The mitochondrial basis of aging. Mol Cell. 2016;61(5):654-66.

34. Debalsi K, Hoff K, Copeland W. Role of the mitochondrial Dna replication machinery in mitochondrial Dna mutagenesis, aging and age-related diseases. Ageing Res Rev. 2017;33:89-104.

35. Bratic A, Larsson $\mathrm{N}$. The role of mitochondria in aging. J Clin Invest. 2013;123(3):951-7.

36. Schon E, Dimauro S, Hirano M. Human mitochondrial Dna: roles of inherited and somatic mutations. Nat Rev Genet. 2012;13(12):878-90.

37. Greaves L, Nooteboom M, Elson J, Tuppen H, Taylor G, Commane D, Arasaradnam R, Khrapko K, Rw T, Kirkwood T, et al. Clonal expansion of early to mid-life mitochondrial Dna point mutations drives mitochondrial dysfunction during human ageing. PLOS Genet. 2014;10(9):E1004620.

38. Ross J, Stewart J, Hagstrom E, Brene S, Mourier A, Coppotelli G, Freyer C, Lagouge M, Hoffer B, Olson L, et al. Germline mitochondrial Dna mutations aggravate ageing and can impair brain development. Nature. 2013; 501(7467):412-5.

39. Wachsmuth M, Hubner A, Li M, Madea B, Stoneking M. Age-related and Heteroplasmy-related variation in human Mtdna copy number. PLoS Genet. 2016;12(3):E1005939.

40. Larsson N. Somatic mitochondrial Dna mutations in mammalian aging. Annu Rev Biochem. 2010;79:683-706.

41. Attardi G, Yoneda M, Chomyn A. Complementation and segregation behavior of disease-causing mitochondrial Dna mutations in cellular model systems. Biochim Biophys Acta. 1995;1271(1):241-8.

42. Diroma M, Calabrese C, Simone D, Santorsola M, Calabrese F, Gasparre G, Attimonelli M. Extraction and annotation of human mitochondrial genomes from 1000 genomes whole exome sequencing data. BMC Genomics. 2014;15(Suppl 3):S2.

43. Lott M, Leipzig J, Derbeneva O, Xie H, Chalkia D, Sarmady M, Procaccio V, Wallace D. Mtdna variation and analysis using Mitomap and Mitomaster. Curr Protoc Bioinformatics. 2013:44:1 23 21-6.

44. Brandon M, Lott M, Nguyen K, Spolim S, Navathe S, Baldi P, Wallace D. Mitomap: a human mitochondrial genome database-2004 update. Nucleic Acids Res. 2005;33(Database Issue):D611-3.

45. Yao Y, Salas A, Logan I, Bandelt H. Mtdna Data Mining In Genbank Needs Surveying. Am J Hum Genet. 2009;85(6):929-33. Author Reply 933

46. Diroma M, Calabrese C, Simone D, Santorsola M, Calabrese F, Gasparre G, Attimonelli M. Extraction and annotation of human mitochondrial genomes from 1000 genomes whole exome sequencing data. BMC Genomics. 2014;15 
47. Genomes Project C, Auton A, Brooks L, Durbin R, Garrison E, Kang H, Korbel J, Marchini J, Mccarthy S, Mcvean G, et al. A global reference for human genetic variation. Nature. 2015;526(7571):68-74.

48. Jaiswal S, Fontanillas P, Flannick J, Manning A, Grauman P, Mar B, Lindsley R, Mermel C, Burtt N, Chavez A, et al. Age-related Clonal Hematopoiesis associated with adverse outcomes. N Engl J Med. 2014;371(26):2488-98.

49. Lo Sardo V, Ferguson W, Erikson G, Topol E, Baldwin K, Torkamani A. Influence of donor age on induced Pluripotent stem cells. Nat Biotechnol. 2017;35(1):69-74.

Submit your next manuscript to BioMed Central and we will help you at every step:

- We accept pre-submission inquiries

- Our selector tool helps you to find the most relevant journal

- We provide round the clock customer support

- Convenient online submission

- Thorough peer review

- Inclusion in PubMed and all major indexing services

- Maximum visibility for your research

Submit your manuscript at www.biomedcentral.com/submit 